\title{
Comparison of prevalence and risk factors of somatization between Chinese health care workers and non-health care workers during COVID-19 outbreak
}

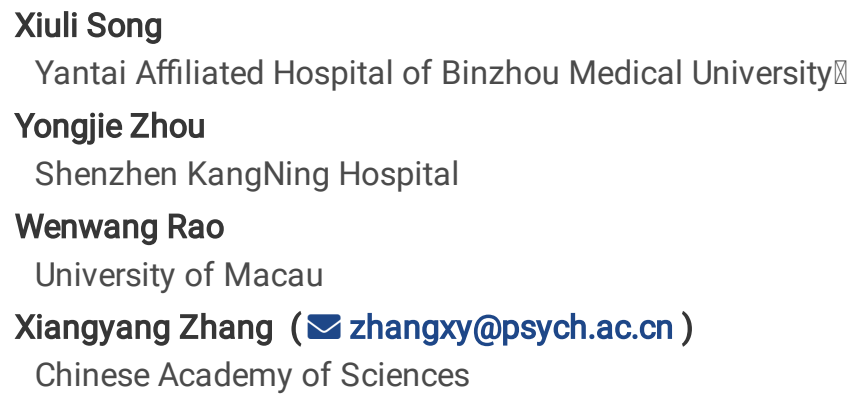

\section{Research Article}

Keywords: COVID-19, Health care workers, Somatization, Risk factors

Posted Date: March 5th, 2021

DOI: https://doi.org/10.21203/rs.3.rs-269527/v1

License: @ (i) This work is licensed under a Creative Commons Attribution 4.0 International License. Read Full License 


\section{Abstract}

Background: This study aimed to compare prevalence and risk factors of somatization (SOM) between health care workers and nonhealth care workers during COVID-19 outbreak in China.

Methods: From 14 February to 29 March 2020, an online survey was performed in both 605 health care workers and 1151 non-health care workers. Based on the somatization dimension score of the Symptom Checklist-90, participants were divided into non-SOM group and SOM group.

Results: Health care workers had higher prevalence rate of SOM $(p<0.001)$ than non-health care workers, with an OR of $1.70(95 \% \mathrm{Cl}$ : $1.22-2.36, p=0.002)$. Multiple logistic regression analysis revealed that in non-health care workers, the risk factors of SOM included other ethnicities, insomnia, and suicide, while in health care workers, the risk factors included working 6-8 hours per day, and working $\geq 10$ hours per day during COVID-19 outbreak.

Conclusions: Our research suggests that both non-health care workers and health care workers have a relatively high prevalence of somatization. However, the related factors for somatization in both groups are significantly different, showing that medical servicerelated factors are associated with somatization in health care workers, while demographic and clinical factors are associated with somatization in non-health care workers.

\section{Background}

The coronavirus disease 2019 (COVID-19) epidemic first occurred in Wuhan city, Hubei province, China, and then spread rapidly nationwide from December 2019 up to date [1]. During the COVID-19 epidemic, a total of 7731 confirmed cases and 170 deaths were reported by 30 January. As the COVID-19 epidemic spread rapidly to all provinces in China within a month, the World Health Organization (WHO) announced the COVID-19 outbreak as a Public Health Emergency of International Concern (PHEIC) (World Health Organization, 2020a) on January 30, 2020 [2]. From then on, the COVID-19 epidemic has been spreading fast all over the world. According to Dr. Tedros, Director-General of WHO, the threat of a global pandemic caused by the coronavirus is now very real. On March 11, 2020, the WHO declared COVID-19 as a pandemic [2].

To efficiently control the COVID-19 outbreak, the Chinese government launched the Public Health Emergency Response (level I) in mainland China on January 29 [3], which means that some practical measures have been implemented, including partial blockades in most cities, community lockdown, cancelation of activities, suspension of most means of transportion, and prohibition of unnecessary gatherings $[2,4,5]$. These measures are aimed at reducing the probability of transmission between infected and uninfected persons [6]. However, the implementation of the above-mentioned measures may also bring some problems, which will have an impact on social norms, interpersonal relationship, the economy and the psychological wellbeing of the population [5]. Previous studies have shown that distress is inevitable if people who suffer from restrictions, loss of daily routines, and lack of interpersonal communication with others can frequently have feelings of boredom, headache, frustration, loneliness and isolation from the world [7-9]. The distress may be exacerbated when people are unable to participate in daily activities for a long time [10].

In addition to isolation, people's mental health is also affected by the rapid spread of the COVID-19 disease, its severity, increased incidences and mortality, lack of effective treatment and vaccines, and the availability of basic resources, such as hand sanitizers, facial masks and digital thermometers. These problems can lead to mental disorders, including depression and anxiety disorders, insomnia and posttraumatic stress disorder (PTSD) [11], which in turn may cause more serious harm than the COVID-19 epidemic itself [3]. Some studies have shown that infectious diseases can bring psychological changes not only to health care workers but also to non-health care workers $[12,13]$, suggesting that COIVD-19 can cause psychological changes $[1,2,14-16]$. For example, one study conducted by Zhang et al. showed that during the outbreak of COVID-19 in China, more than 33\% of medical staffs developed symptoms of insomnia from January 29 to February 3[17]. Another study performed by Lai et al. in China indicated that from January 29 to February 3, the prevalence rates of distress symptoms, depression, anxiety and insomnia were $71.5 \%, 50.4 \%, 44.6 \%, 34.0 \%$, respectively [18]. Tian and his colleagues found that from January 31 to February 2, more than $70 \%$ of participants had moderate to severe psychological symptoms, especially obsessive compulsive disorder, phobic anxiety, interpersonal sensitivity and psychiatric disorders in the Chinese general population [2]. Chew et al. found that physical symptoms were associated with higher average scores calculated by the Impact of Events Scale-Revised (IES-R) and Depression Anxiety Stress Scales (DASS-21) for healthcare workers in Singapore and India from February 19 to April 17, 2020 [16].

Page 2/18 
To date, there have been few studies on the comparison of psychological symptoms between non-health care workers and health care workers during the COVID-19 outbreak in China and other countries. For instance, Tian et al. found that there was a significant difference in the somatization score of SCL-90 between health care workers and general population ( $1.81 \pm 0.69 \mathrm{vs} 1.37 \pm 0.48, p=$ 0.001 [ [2]. Chew et al. reported that there was a significant association between the risk of physical symptoms and psychological distress among health care workers during the COVID-19 epidemic [16].

Therefore, the main purposes of this study were to (1) compare the differences in demographic and clinical data between non-health care workers and health care workers; and (2) to explore the risk factors of somatization severity shown on SCL-90 scale between health care workers and non-health care workers.

\section{Materials And Methods}

\section{Study design and participants}

This is an online epidemiological survey using a self-administered questionnaire during the COVID-19 epidemic. The data were conducted by the "Wenjuanxing" Survey Platform in China from February 14 to March 29, 2020. This study recruited 1,756 participants including 1,151 non-health care workers and 605 health care workers. This study protocol was reviewed and approved by the Institute of Psychology, Chinese Academy of Sciences. Ethical approval was

conducted in accordance with the latest version of the Helsinki Declaration (line 96-98). All participants received an electronic informed consent form and then signed the form to participate in the study.

\section{Assessment}

Demographic data, self-designed questionnaire related to the COVID-19 outbreak, and the SOM dimension of SCL-90 scale, the Insomnia Severity Index (ISI) scale, and suicide module of Mini International Neuropsychiatric Interview (MINI) were obtained through the "Wenjuanxing" Survey Platform.

SCL-90 was used to measure psychological distress and psychopathological symptoms [19]. The 90-item self-reported symptom survey is categorized into nine dimensions: Somatization (SOM), Obsessive-Compulsive (OC), Interpersonal Sensitivity (IS), Depression (DEP), Anxiety (ANX), Hostility (HOS), Phobic Anxiety (PHOB), Paranoid Ideation (PAR), and Psychoticism (PSY). We selected the SOM dimension (12 items) of the SCL-90 to assess the severity of physical discomfort. Each item is scored on a 1-5 scale, and the total score ranges from 12 to 60 . The total score is divided into different degrees of somatic discomfort symptoms: no somatic discomfort (<24, i.e., non-SOM group), as well as minimal, moderate and severe somatic discomfort ( $\geq 24$, i.e., SOM group).

The Insomnia Severity Index (ISI) was performed to assess the severity of insomnia symptoms [20]. Each item is graded on a scale of $0-4$, and the total score of the 7-item ISI ranges from 0 to 28 . The total score is categorized into four different groups: no insomnia $(0-7)$, mild (8-14), moderate (15-21), and severe (22-28).

Mini International Neuropsychiatric Interview (MINI) was designed as a brief structured interview for major Axis I psychiatric disorders in DSM-IV and ICD-10 [21]. We selected the suicide module (7 items) of the MINI to assess the severity of suicide symptoms. The total score is classified into: no (0), mild (1-5), moderate (6-9), and severe $(\geq 10)$.

\section{Statistical analysis}

Data were analyzed using SPSS statistical software for Windows (version 22.0., IBM Corp.). The categorized variables between the two groups were analyzed by chi-square test. Kolmogorov-Smirnov single sample test was used to assess the normality of continuous variables. For the data of normal distribution, the independent Student's t-test was used to compare the differences between two groups. For the data of non-normal distribution, the median and the interquartile ranges (IQRs) were presented and the Wilcoxon test (Mann-Whitney test) was used to compare the differences between two groups. Demographic data and clinical symptoms were analyzed with $2 \times 2$ ANOVA representing the between factors of group (non-health care workers vs. health care workers) and diagnose (non-SOM group vs. SOM group). Finally, multiple logistic regression analysis with the forward stepwise method was carried out to examine potential risk factors of SOM in different groups. $P$-value $<0.05$ with 2-tailed tests was regarded as statistical significance. 


\section{Results}

\section{Prevalence of somatization between non-health care workers and health care workers}

A total of 1,756 participants completed the survey, including 1,151 (65.55\%) non-health care workers and $605(34.45 \%)$ health care workers.

The prevalence rate of SOM in health care workers was $9.59 \%$, which was significantly higher than that in non-health care workers (5.45\%), with an OR of 1.70 (95\% confidence interval: $\left.1.22-2.36 ; \chi^{2}=9.80, d f=1, p=0.002\right)$. After controlling for the sociodemographic confounders, such as gender, age, ethnicity, education, marital status, living situation and BMI, logistic regression analysis showed that there was still a significant difference, with an adjusted OR of 1.66 ( $\left.95 \% \mathrm{Cl}: 1.15-2.39 ; \chi^{2}=7.26, p=0.007\right)$. Further, there were significant differences between non-health care workers and health care workers in terms of sex, age, body mass index (BMI), ethnicity, marital status, education level, living status, relatives and friends infected with COVID-19, experience SARS personally, income level, economic loss, medical disease, somatization, insomnia, drinking (all $p<0.05)($ Table 1$)$. 
Table 1

Demographic data and clinical symptoms between non-health care workers and health care workers.

\begin{tabular}{|c|c|c|c|c|}
\hline Variables & $\begin{array}{l}\text { Non-health care workers }(n= \\
1151)\end{array}$ & $\begin{array}{l}\text { Health care workers }(n= \\
605)\end{array}$ & $z / x^{2}$ & $p$ \\
\hline \multicolumn{5}{|l|}{ Sex, n (\%) } \\
\hline Men & 356(30.93) & 114(18.84) & 29.56 & $\begin{array}{l}< \\
0.001\end{array}$ \\
\hline Women & 795(69.07) & 491(81.16) & & \\
\hline Age (years), Median (IQR) & $22(21-37)$ & $35(30-41)$ & -15.81 & $<.001$ \\
\hline BMI (kg/m²), Median (IQR) & $21.51(19.72-24.16)$ & $22.03(20.22-24.35)$ & -2.57 & 0.01 \\
\hline \multicolumn{5}{|l|}{ Ethnicity, n (\%) } \\
\hline Han & 1092(94.87) & $558(92.23)$ & 4.88 & 0.03 \\
\hline Others & $59(5.13)$ & 47(7.77) & & \\
\hline \multicolumn{5}{|l|}{ Marital status, n (\%) } \\
\hline Single & $689(59.86)$ & $122(20.17)$ & 260.30 & $<$. \\
\hline Married or cohabiting & $413(35.88)$ & 456(75.37) & & \\
\hline Divorced, separated or widowed & $49(4.26)$ & $27(4.46)$ & & \\
\hline \multicolumn{5}{|l|}{ Education level, n (\%) } \\
\hline High school or below & $148(12.86)$ & $14(2.31)$ & 110.32 & <. \\
\hline Junior college and Bachelor's degree & $897(77.93)$ & $445(73.55)$ & & \\
\hline Master's degree or above & $106(9.21)$ & $146(24.13)$ & & \\
\hline \multicolumn{5}{|l|}{ Occupation, n (\%) } \\
\hline Student & $586(50.91)$ & NA & & \\
\hline Professional & $339(29.45)$ & NA & & \\
\hline Teacher & $57(4.95)$ & NA & & \\
\hline Others & 189(16.42) & NA & & \\
\hline Doctor & NA & 208(34.38) & & \\
\hline Nurse & NA & $333(55.04)$ & & \\
\hline Medical technician & NA & $64(10.58)$ & & \\
\hline \multicolumn{5}{|l|}{ Living situation, n (\%) } \\
\hline Wuhan & $17(1.48)$ & $28(4.63)$ & 37.98 & $\begin{array}{l}< \\
0.001\end{array}$ \\
\hline Hubei province outside Wuhan & $14(1.22)$ & $29(4.79)$ & & \\
\hline Outside Hubei province & 1120(97.31) & $548(90.58)$ & & \\
\hline \multicolumn{5}{|l|}{ Length of service (years), n (\%) } \\
\hline$\leq 5$ & NA & $110(18.18)$ & & \\
\hline $6-10$ & NA & $162(26.78)$ & & \\
\hline$\geq 10$ & NA & 194(32.07) & & \\
\hline
\end{tabular}




\begin{tabular}{|c|c|c|c|c|}
\hline Variables & $\begin{array}{l}\text { Non-health care workers }(n= \\
1151)\end{array}$ & $\begin{array}{l}\text { Health care workers }(n= \\
605)\end{array}$ & $\mathrm{Z} / \mathrm{X}^{2}$ & $p$ \\
\hline$\geq 20$ & NA & 139(22.98) & & \\
\hline \multicolumn{5}{|c|}{$\begin{array}{l}\text { Working hours per day during COVID-19 } \\
\text { outbreak, } \mathrm{n}(\%)\end{array}$} \\
\hline $4-6$ & NA & $40(6.61)$ & & \\
\hline $6-8$ & NA & 243(40.17) & & \\
\hline $8-10$ & NA & $268(44.30)$ & & \\
\hline$\geq 10$ & NA & $54(8.93)$ & & \\
\hline \multicolumn{5}{|c|}{$\begin{array}{l}\text { Relatives and friends infected with coronavirus, } \\
\mathrm{n}(\%)\end{array}$} \\
\hline No & 1143(99.30) & 592(97.85) & 7.09 & 0.01 \\
\hline Yes & $8(0.70)$ & $13(2.15)$ & & \\
\hline \multicolumn{5}{|c|}{ Experience SARS personally, $\mathrm{n}(\%)$} \\
\hline No & $594(51.61)$ & $343(56.69)$ & 4.12 & 0.04 \\
\hline Yes & $557(48.39)$ & 262(43.31) & & \\
\hline \multicolumn{5}{|c|}{ Income (ten thousand), n (\%) } \\
\hline$\leq 8$ & $488(42.40)$ & 105(17.36) & 111.35 & $<.001$ \\
\hline $8-30$ & $539(46.83)$ & $402(66.45)$ & & \\
\hline$\geq 30$ & 124(10.77) & $98(16.20)$ & & \\
\hline \multicolumn{5}{|c|}{ Economic loss (ten thousand), n (\%) } \\
\hline$\leq 3$ & 791(68.72) & $323(53.39)$ & 56.79 & $<.001$ \\
\hline $3-10$ & $82(7.12)$ & $89(14.71)$ & & \\
\hline$\geq 10$ & 82(7.12) & $83(13.72)$ & & \\
\hline Unknown & 202(17.55) & $110(18.18)$ & & \\
\hline \multicolumn{5}{|l|}{ Medical illness, n (\%) } \\
\hline No & 1002(87.05) & 468(77.36) & 27.36 & $<.001$ \\
\hline Yes & 149(12.95) & 137(22.64) & & \\
\hline \multicolumn{5}{|l|}{ Somatization, n (\%) } \\
\hline No $(<24)$ & 1108(96.26) & $547(90.41)$ & 11.12 & $\begin{array}{l}< \\
0.001\end{array}$ \\
\hline Yes $(\geq 24)$ & $63(5.45)$ & $58(9.59)$ & & \\
\hline Insomnia, Median (IQR) & $3(0-7)$ & $5(1-9)$ & -4.44 & $<.001$ \\
\hline Suicide, Median (IQR) & $0(0-0)$ & $0(0-0)$ & -0.87 & 0.38 \\
\hline \multicolumn{5}{|l|}{ Smoking, n (\%) } \\
\hline No & 1032(89.66) & $550(90.91)$ & 0.94 & 0.62 \\
\hline Yes & $82(7.12)$ & $40(6.61)$ & & \\
\hline
\end{tabular}




\begin{tabular}{|c|c|c|c|c|}
\hline Variables & $\begin{array}{l}\text { Non-health care workers }(n= \\
1151)\end{array}$ & $\begin{array}{l}\text { Health care workers }(n= \\
605)\end{array}$ & $\mathrm{Z} / \mathrm{X}^{2}$ & $p$ \\
\hline smoking cessation & $37(3.21)$ & $15(2.48)$ & & \\
\hline \multicolumn{5}{|l|}{ Drinking, n (\%) } \\
\hline No & $884(76.80)$ & $460(76.03)$ & 7.21 & 0.03 \\
\hline Yes & 212(18.42) & $130(21.49)$ & & \\
\hline Abstinence & $55(4.78)$ & $15(2.48)$ & & \\
\hline
\end{tabular}

Comparison of SCL-90 SOM dimension subscale between non-health care workers and health care workers

Health care workers had higher SOM total score $(p<0.001)$ than non-health care workers. Each item score of SOM was significantly higher in health care workers than that in non-health care workers $(p<0.001 \sim p<0.05)($ Table 2$)$.

Table 2

Comparison of SCL-90 SOM dimension subscale between non-health care workers and health care workers

\begin{tabular}{|llll|}
\hline Variables & $\begin{array}{l}\text { Non-health care workers } \\
(\mathbf{n = 1 1 5 1 )}\end{array}$ & $\begin{array}{l}\text { health care workers } \\
(\mathbf{n = 6 0 5})\end{array}$ & $<0.001$ \\
\hline total scores & $14.0(12.0-17.0)$ & $16.0(14.0-20.0)$ & $<0.001$ \\
\hline Headaches & $1.0(1.0-2.0)$ & $2.0(1.0-2.0)$ & $<0.001$ \\
\hline Faintness or dizziness & $1.0(1.0-1.0)$ & $1.0(1.0-2.0)$ & 0.003 \\
\hline Pains in heart or chest & $1.0(1.0-1.0)$ & $1.0(1.0-1.0)$ & $<0.001$ \\
\hline Pains in lower back & $1.0(1.0-2.0)$ & $2.0(1.0-2.0)$ & $<0.001$ \\
\hline Nausea or upset stomach & $1.0(1.0-2.0)$ & $1.0(1.0-2.0)$ & $<0.001$ \\
\hline Soreness of your muscles & $1.0(1.0-2.0)$ & $2.0(1.0-2.0)$ & $<0.001$ \\
\hline Trouble getting your breath & $1.0(1.0-1.0)$ & $1.0(1.0-1.0)$ & $<0.001$ \\
\hline Hot or cold spells & $1.0(1.0-1.0)$ & $1.0(1.0-1.0)$ & 0.02 \\
\hline Numbness or tingling in parts of your body & $1.0(1.0-1.0)$ & $1.0(1.0-1.0)$ & 0.046 \\
\hline A lump in your throat & $1.0(1.0-1.0)$ & $1.0(1.0-1.5)$ & $<0.001$ \\
\hline Feeling weak in parts of your body & $1.0(1.0-2.0)$ & $1.0(1.0-2.0)$ & $<0.001$ \\
\hline Heavy feelings in your arms or legs & $1.0(1.0-1.0)$ & $1.0(1.0-2.0)$ & \\
\hline
\end{tabular}

Comparison of demographic data and clinical symptoms by group and diagnose

As shown in Table 3, two-way ANOVA showed that there were significant effects of group on age, marital status, education level, living status, income level, economic loss, insomnia, and suicide (all $p<0.05$ ). There were significant effects of diagnose on age, experience SARS personally, medical illness, insomnia, suicide, and drinking (all $p<0.05$ ). Also, there were significant group $\times$ diagnose effects on sex, medical illness, insomnia, and suicide $(p<0.05)$. 
Table 3

Comparison of demographic data and clinical symptoms by group and diagnose.

\begin{tabular}{|c|c|c|c|c|c|c|c|c|c|c|}
\hline \multirow[t]{2}{*}{ variables } & \multicolumn{2}{|c|}{ Non-health care workers } & \multicolumn{2}{|c|}{ health care workers } & \multicolumn{2}{|l|}{ Group } & \multicolumn{2}{|c|}{ Diagnose } & \multicolumn{2}{|c|}{ Group×Diagnose } \\
\hline & $\begin{array}{l}\text { Non-SOM } \\
\text { group }\end{array}$ & $\begin{array}{l}\text { SOM } \\
\text { group }\end{array}$ & $\begin{array}{l}\text { Non-SOM } \\
\text { group }\end{array}$ & $\begin{array}{l}\text { SOM } \\
\text { group }\end{array}$ & $\mathrm{F}$ & $p$ & $\mathrm{~F}$ & $p$ & $\mathrm{~F}$ & $p$ \\
\hline & $(n=1065)$ & $(n=86)$ & $(n=532)$ & $(n=73)$ & & & & & & \\
\hline Sex, n (\%) & & & & & 2.94 & 0.09 & 3.44 & 0.06 & 4.24 & 0.04 \\
\hline Men & $330(30.99)$ & $26(30.23)$ & $91(17.11)$ & $23(31.51)$ & & & & & & \\
\hline Women & $735(69.01)$ & $60(69.77)$ & $441(82.89)$ & $50(68.49)$ & & & & & & \\
\hline Age (years) & $28.43 \pm 0.30$ & $\begin{array}{l}31.17 \pm \\
1.04\end{array}$ & $\begin{array}{l}35.67 \pm \\
0.42\end{array}$ & $\begin{array}{l}36.53 \pm \\
1.13\end{array}$ & 60.17 & $<.001$ & 4.93 & 0.03 & 1.34 & 0.25 \\
\hline $\mathrm{BMI}\left(\mathrm{kg} / \mathrm{m}^{2}\right)$ & $22.20 \pm 0.11$ & $\begin{array}{l}22.41 \pm \\
0.39\end{array}$ & $\begin{array}{l}22.61 \pm \\
0.16\end{array}$ & $\begin{array}{l}22.94 \pm \\
0.42\end{array}$ & 2.41 & 0.12 & 0.83 & 0.36 & 0.03 & 0.85 \\
\hline Ethnicity, n (\%) & & & & & 0.02 & 0.90 & 0.23 & 0.63 & 3.18 & 0.08 \\
\hline Han & 1014(95.21) & $78(90.70)$ & 489(91.92) & $69(94.52)$ & & & & & & \\
\hline Others & $51(4.79)$ & $8(9.30)$ & $43(8.08)$ & $4(5.48)$ & & & & & & \\
\hline $\begin{array}{l}\text { Marital status, } \mathrm{n} \\
(\%)\end{array}$ & & & & & 68.93 & $<.001$ & 2.8 & 0.10 & 0.24 & 0.63 \\
\hline Single & 648(60.85) & $41(47.67)$ & $111(20.86)$ & 11(15.07) & & & & & & \\
\hline $\begin{array}{l}\text { Married or } \\
\text { cohabiting }\end{array}$ & $369(34.65)$ & $44(51.16)$ & $397(74.62)$ & $59(80.82)$ & & & & & & \\
\hline $\begin{array}{l}\text { Divorced, } \\
\text { separated or } \\
\text { widowed }\end{array}$ & $48(4.51)$ & $1(1.16)$ & $24(4.51)$ & $3(4.11)$ & & & & & & \\
\hline $\begin{array}{l}\text { Education level, } \mathrm{n} \\
(\%)\end{array}$ & & & & & 47.28 & $<.001$ & 0.64 & 0.42 & 0.19 & 0.66 \\
\hline $\begin{array}{l}\text { High school or } \\
\text { below }\end{array}$ & $134(12.58)$ & 14(16.28) & $13(2.44)$ & $1(1.37)$ & & & & & & \\
\hline $\begin{array}{l}\text { Junior college and } \\
\text { Bachelor's degree }\end{array}$ & $832(78.12)$ & $65(75.58)$ & $389(73.12)$ & $56(76.71)$ & & & & & & \\
\hline $\begin{array}{l}\text { Master's degree or } \\
\text { above }\end{array}$ & $99(9.30)$ & $7(8.14)$ & $13(2.44)$ & $1(1.37)$ & & & & & & \\
\hline $\begin{array}{l}\text { Living situation, } \mathrm{n} \\
(\%)\end{array}$ & & & & & 7.94 & 0.005 & 3.91 & 0.05 & 0.73 & 0.39 \\
\hline Wuhan & $17(1.60)$ & $0(0.00)$ & $27(5.08)$ & $1(1.37)$ & & & & & & \\
\hline $\begin{array}{l}\text { Hubei province } \\
\text { outside Wuhan }\end{array}$ & $13(1.22)$ & $1(1.16)$ & $26(4.89)$ & $3(4.11)$ & & & & & & \\
\hline $\begin{array}{l}\text { Outside Hubei } \\
\text { province }\end{array}$ & 1035(97.18) & $85(98.84)$ & $479(90.04)$ & $69(94.52)$ & & & & & & \\
\hline $\begin{array}{l}\text { Relatives and friend } \\
\text { coronavirus, } \mathrm{n}(\%)\end{array}$ & infected with & & & & 2.49 & 0.12 & 0.81 & 0.37 & 0.01 & 0.94 \\
\hline No & 1057(99.25) & $86(100)$ & $520(97.74)$ & $72(98.63)$ & & & & & & \\
\hline Yes & $8(0.75)$ & $0(0.00)$ & $12(2.26)$ & $1(1.37)$ & & & & & & \\
\hline $\begin{array}{l}\text { Experience SARS } \\
\text { personally, } \mathrm{n}(\%)\end{array}$ & & & & & 1.28 & 0.26 & 4.62 & 0.03 & 0.05 & 0.82 \\
\hline No & $556(52.21)$ & $38(44.19)$ & $308(57.89)$ & $35(47.95)$ & & & & & & \\
\hline
\end{tabular}




\begin{tabular}{|c|c|c|c|c|c|c|c|c|c|c|}
\hline \multirow{2}{*}{$\begin{array}{l}\text { variables } \\
\text { Yes }\end{array}$} & \multicolumn{2}{|c|}{ Non-health care workers } & \multicolumn{2}{|c|}{ health care workers } & \multicolumn{2}{|l|}{ Group } & \multicolumn{2}{|c|}{ Diagnose } & \multicolumn{2}{|c|}{ Group×Diagnose } \\
\hline & $509(47.79)$ & $48(55.81)$ & $224(42.11)$ & $38(52.05)$ & & & & & & \\
\hline $\begin{array}{l}\text { Income (ten } \\
\text { thousand), n (\%) }\end{array}$ & & & & & 42.32 & $\begin{array}{l}< \\
0.001\end{array}$ & 1.68 & 0.20 & 1.02 & 0.31 \\
\hline$\leq 8$ & $448(42.07)$ & $40(46.51)$ & $97(18.23)$ & $8(10.96)$ & & & & & & \\
\hline $8-30$ & $507(47.61)$ & $32(37.21)$ & $352(66.17)$ & $50(68.49)$ & & & & & & \\
\hline$\geq 30$ & 110(10.33) & $14(16.28)$ & $83(15.60)$ & $15(20.55)$ & & & & & & \\
\hline $\begin{array}{l}\text { Economic loss } \\
\text { (ten thousand), n } \\
(\%)\end{array}$ & & & & & 9.9 & 0.002 & 0.21 & 0.65 & 0.77 & 0.38 \\
\hline$\leq 3$ & $734(68.92)$ & $57(66.28)$ & 286(53.76) & $37(50.68)$ & & & & & & \\
\hline $3-10$ & $72(6.76)$ & $10(11.63)$ & $79(14.85)$ & $10(13.70)$ & & & & & & \\
\hline$\geq 10$ & $74(6.95)$ & $8(9.30)$ & $74(13.91)$ & $9(12.33)$ & & & & & & \\
\hline Unknown & 185(17.37) & $11(12.79)$ & $93(17.48)$ & $17(23.29)$ & & & & & & \\
\hline $\begin{array}{l}\text { Medical illness, n } \\
(\%)\end{array}$ & & & & & 0.07 & 0.80 & 4.85 & 0.03 & 12.19 & $\begin{array}{l}< \\
0.001\end{array}$ \\
\hline No & 124(11.64) & $25(29.07)$ & $123(23.12)$ & 14(19.18) & & & & & & \\
\hline Yes & $941(88.36)$ & 61(70.93) & 409(76.88) & $59(80.82)$ & & & & & & \\
\hline Insomnia & $4.52 \pm 0.17$ & $\begin{array}{l}11.15 \pm \\
0.60\end{array}$ & $6.22 \pm 0.24$ & $\begin{array}{l}6.44 \pm \\
0.65\end{array}$ & 10.31 & 0.001 & 53.35 & $<001$ & 46.77 & $\begin{array}{l}< \\
0.001\end{array}$ \\
\hline Suicide & $0.71 \pm 0.12$ & $\begin{array}{l}4.41 \pm \\
0.43\end{array}$ & $1.07 \pm 0.17$ & $\begin{array}{l}0.81 \pm \\
0.46\end{array}$ & 23.82 & $<0.001$ & 26.92 & $<0.001$ & 35.65 & $\begin{array}{l}< \\
0.001\end{array}$ \\
\hline Smoking, n (\%) & & & & & 0.12 & 0.73 & 0.67 & 0.42 & 0.11 & 0.74 \\
\hline No & 955(89.67) & 77(89.53) & 486(91.35) & $64(87.67)$ & & & & & & \\
\hline Yes & 77(7.23) & $5(5.81)$ & $33(6.20)$ & $7(9.59)$ & & & & & & \\
\hline smoking cessation & $33(3.10)$ & $4(4.65)$ & $13(2.44)$ & $2(2.74)$ & & & & & & \\
\hline Drinking, n (\%) & & & & & 1.08 & 0.30 & 5.66 & 0.02 & 0.53 & 0.47 \\
\hline No & $825(77.46)$ & $59(68.60)$ & 409(76.88) & $51(69.86)$ & & & & & & \\
\hline Yes & 193(18.12) & 19(22.09) & $110(20.68)$ & $20(27.40)$ & & & & & & \\
\hline Abstinence & $47(4.41)$ & $8(9.30)$ & $13(2.44)$ & $2(2.73)$ & & & & & & \\
\hline
\end{tabular}


Table 4

Demographic data and clinical symptoms between non-SOM and SOM groups of non-health care workers

\begin{tabular}{|c|c|c|c|c|}
\hline & \multicolumn{4}{|c|}{ Non-health care workers } \\
\hline & Non-SOM group & SOM group & $z / x^{2}$ & $p$ \\
\hline & $(n=1065)$ & $(n=86)$ & & \\
\hline \multicolumn{5}{|l|}{ Sex, n (\%) } \\
\hline Men & $330(30.99)$ & $26(30.23)$ & 0.02 & 0.88 \\
\hline Women & 735(69.01) & $60(69.77)$ & & \\
\hline Age (years), Median (IQR) & $22(21-37)$ & $30.50(22-38)$ & -2.91 & $<0.001$ \\
\hline BMI (kg/m2), Median (IQR) & 21.51(19.71-24.17) & $21.50(20.02-23.89)$ & -0.74 & 0.46 \\
\hline \multicolumn{5}{|l|}{ Ethnicity, n (\%) } \\
\hline Han & 1014(95.21) & 78(90.70) & 3.33 & 0.07 \\
\hline Other & $51(4.79)$ & $8(9.30)$ & & \\
\hline \multicolumn{5}{|l|}{ Marital status, n (\%) } \\
\hline Single & $648(60.85)$ & $41(47.67)$ & 10.45 & 0.005 \\
\hline Married or cohabiting & $369(34.65)$ & $44(51.16)$ & & \\
\hline Divorced, separated or widowed & $48(4.51)$ & $1(1.16)$ & & \\
\hline \multicolumn{5}{|l|}{ Education level, n (\%) } \\
\hline High school or below & 134(12.58) & $14(16.28)$ & 1.03 & 0.60 \\
\hline Junior college and Bachelor's degree & $832(78.12)$ & $65(75.58)$ & & \\
\hline Master's degree or above & $99(9.30)$ & 7(8.14) & & \\
\hline \multicolumn{5}{|l|}{ Occupation, n (\%) } \\
\hline Student & $539(50.61)$ & $30(34.88)$ & 7.92 & 0.048 \\
\hline Professional & $306(28.73)$ & $33(38.37)$ & & \\
\hline Teacher & $51(4.79)$ & $5(5.81)$ & & \\
\hline Other & 169(15.87) & 18(20.93) & & \\
\hline \multicolumn{5}{|l|}{ Living situation, n (\%) } \\
\hline Wuhan & $17(1.60)$ & $0(0.00)$ & 1.40 & 0.50 \\
\hline Hubei province outside Wuhan & $13(1.22)$ & $1(1.16)$ & & \\
\hline Outside Hubei province & 1035(97.18) & $85(98.84)$ & & \\
\hline \multicolumn{5}{|c|}{ Relatives and friends infected with coronavirus, $\mathrm{n}(\%)$} \\
\hline No & 1057(99.25) & $86(100)$ & 0.65 & 0.42 \\
\hline Yes & $8(0.75)$ & $0(0.00)$ & & \\
\hline \multicolumn{5}{|l|}{ Experience SARS personally, $\mathrm{n}(\%)$} \\
\hline No & $556(52.21)$ & $38(44.19)$ & 2.05 & 0.15 \\
\hline Yes & $509(47.79)$ & $48(55.81)$ & & \\
\hline
\end{tabular}




\begin{tabular}{|c|c|c|c|c|}
\hline Income, n (\%) & \multicolumn{4}{|c|}{ Non-health care workers } \\
\hline$\leq 8$ & $448(42.07)$ & $40(46.51)$ & 4.82 & 0.09 \\
\hline $8-30$ & $507(47.61)$ & $32(37.21)$ & & \\
\hline$\geq 30$ & $110(10.33)$ & $14(16.28)$ & & \\
\hline \multicolumn{5}{|l|}{ Economic loss, n (\%) } \\
\hline$\leq 3$ & $734(68.92)$ & $57(66.28)$ & 4.33 & 0.23 \\
\hline $3-10$ & $72(6.76)$ & $10(11.63)$ & & \\
\hline$\geq 10$ & $74(6.95)$ & $8(9.30)$ & & \\
\hline Unknown & 185(17.37) & $11(12.79)$ & & \\
\hline \multicolumn{5}{|l|}{ Medical illness, n (\%) } \\
\hline Yes & $124(11.64)$ & $25(29.07)$ & 21.44 & $<0.001$ \\
\hline No & $941(88.36)$ & $61(70.93)$ & & \\
\hline Insomnia, Median (IQR) & $3(0-7)$ & $12(6-15)$ & -8.86 & $<0.001$ \\
\hline Suicide, Median (IQR) & $0(0-0)$ & $0(0-4)$ & -8.72 & $<0.001$ \\
\hline \multicolumn{5}{|l|}{ Smoking, n (\%) } \\
\hline No & $955(89.67)$ & $77(89.53)$ & 0.82 & 0.66 \\
\hline Yes & $77(7.23)$ & $5(5.81)$ & & \\
\hline Smoking cessation & $33(3.10)$ & $4(4.65)$ & & \\
\hline \multicolumn{5}{|l|}{ Drinking, n (\%) } \\
\hline No & $825(77.46)$ & $59(68.60)$ & 5.48 & 0.07 \\
\hline Yes & 193(18.12) & 19(22.09) & & \\
\hline Abstinence & $47(4.41)$ & $8(9.30)$ & & \\
\hline
\end{tabular}

\section{Demographic data and clinical symptoms between non-SOM and SOM groups of non-health care workers}

All non-health care workers were divided into two groups: non-SOM group (SOM total score <24) and SOM group (SOM total score $\geq$ 24). There were significant differences in age, marital status, occupation, medical illness, insomnia, and suicide between two groups (Tabel 4). Multiple logistic regression showed that other ethnicities (non-Han Chinese) $(\mathrm{OR}=2.45, p=0.04)$, insomnia $(\mathrm{OR}=1.16, p<$ $0.001)$, suicide $(\mathrm{OR}=1.08, p<0.001)$ and being single $(\mathrm{OR}=0.52, p=0.01)$ were associated with the SOM of non-health care workers (Table 5). 
Table 5

Multiple logistic regression analysis of SOM-related factors of non-health care workers.

\begin{tabular}{|llllll|}
\hline Variable & B & SE & $\boldsymbol{p}$ & OR & $95 \% \mathrm{Cl}$ \\
\hline Ethnicity (ref: Han) & 0.90 & 0.44 & 0.04 & 2.45 & $1.04-5.78$ \\
\hline Insomnia & 0.15 & 0.02 & $<0.001$ & 1.16 & $1.12-1.20$ \\
\hline Suicide & 0.07 & 0.02 & $<0.001$ & 1.08 & $1.04-1.12$ \\
\hline Marital status (ref: Married or cohabiting) & & & 0.01 & & \\
\hline Single & -0.66 & 0.25 & 0.01 & 0.52 & $0.32-0.84$ \\
\hline Divorced, separated or widowed & -1.99 & 1.05 & 0.06 & 0.14 & $0.02-1.08$ \\
\hline Constant & -6.27 & 1.16 & $<0.001$ & 0.002 & \\
\hline
\end{tabular}

\section{Demographic data and clinical symptoms in non-SOM and SOM groups of health care workers}

All health care workers were divided into two groups: non-SOM group and SOM group.

There were significant differences in sex, and working hours per day during COVID-19 outbreak between non-SOM group and SOM group of health care workers (Table 6). Further multiple logistic regression showed that women $(\mathrm{OR}=0.46, p=0.01)$, working $6-8$ hours per day during COVID-19 outbreak $(\mathrm{OR}=14.87, p=0.01)$, and working $\geq 10$ hours per day during COVID-19 outbreak $(\mathrm{OR}=11.07$, $p=0.02$ ) were independently associated with the SOM of health care workers (Tabel 7). 
Table 6

Demographic data and clinical symptoms between non-SOM and SOM groups of health care workers.

\begin{tabular}{|c|c|c|c|c|}
\hline & \multicolumn{4}{|l|}{ Health care workers } \\
\hline & Non-SOM group & SOM group & $\mathrm{Z} / \mathrm{X}^{2}$ & $p$ \\
\hline & $(n=532)$ & $(n=73)$ & & \\
\hline \multicolumn{5}{|l|}{ Sex, n (\%) } \\
\hline Men & $91(17.11)$ & 23(31.51) & 8.71 & 0.003 \\
\hline Women & $441(82.89)$ & $50(68.49)$ & & \\
\hline Age (years), Median (IQR) & $35(30-41)$ & $36(30-44)$ & -0.93 & 0.35 \\
\hline BMI (kg/m2), Median (IQR) & $22.03(20.20-24.43)$ & $22.60(20.71-24.60)$ & -0.82 & 0.41 \\
\hline \multicolumn{5}{|l|}{ Ethnicity, n (\%) } \\
\hline Han & 489(91.92) & $69(94.52)$ & 0.61 & 0.44 \\
\hline Other & 43(8.08) & $4(5.48)$ & & \\
\hline \multicolumn{5}{|l|}{ Marital status, n (\%) } \\
\hline Single & 111(20.86) & 11(15.07) & 1.42 & 0.49 \\
\hline Married or cohabiting & $397(74.62)$ & $59(80.82)$ & & \\
\hline Divorced, separated or widowed & $24(4.51)$ & $3(4.11)$ & & \\
\hline \multicolumn{5}{|l|}{ Education level, n (\%) } \\
\hline High school or below & $13(2.44)$ & $1(1.37)$ & 0.60 & 0.74 \\
\hline Junior college and Bachelor's degree & $389(73.12)$ & $56(76.71)$ & & \\
\hline Master's degree or above & $130(24.44)$ & 16(21.92) & & \\
\hline \multicolumn{5}{|l|}{ Occupation, n (\%) } \\
\hline Doctor & 180(33.83) & $28(38.36)$ & 2.44 & 0.30 \\
\hline Nurse & 292(55.89) & $41(56.16)$ & & \\
\hline Medical technician & $60(11.28)$ & $4(5.48)$ & & \\
\hline \multicolumn{5}{|l|}{ Living situation, n (\%) } \\
\hline Wuhan & $27(5.08)$ & $1(1.37)$ & 2.13 & 0.35 \\
\hline Hubei province outside Wuhan & $26(4.89)$ & $3(4.11)$ & & \\
\hline Outside Hubei province & $479(90.04)$ & $69(94.52)$ & & \\
\hline \multicolumn{5}{|l|}{ Length of service, n (\%) } \\
\hline$\leq 5$ & $99(18.61)$ & $11(15.07)$ & 1.65 & 0.65 \\
\hline $6-10$ & 144(27.07) & $18(24.66)$ & & \\
\hline$\geq 10$ & 166(31.20) & $28(38.36)$ & & \\
\hline$\geq 20$ & 123(23.12) & 16(21.92) & & \\
\hline \multicolumn{5}{|c|}{ Working hours per day during COVID-19 outbreak, n (\%) } \\
\hline $4-6$ & $31(5.83)$ & $9(12.33)$ & 15.38 & 0.002 \\
\hline $6-8$ & 222(41.73) & 21(28.77) & & \\
\hline
\end{tabular}




\begin{tabular}{|c|c|c|c|c|}
\hline \multirow[b]{2}{*}{$8-10$} & \multicolumn{4}{|c|}{ Health care workers } \\
\hline & $226(42.48)$ & $42(57.53)$ & & \\
\hline$\geq 10$ & $53(9.96)$ & $1(1.37)$ & & \\
\hline \multicolumn{5}{|c|}{ Relatives and friends infected with coronavirus, $\mathrm{n}(\%)$} \\
\hline No & $520(97.74)$ & $72(98.63)$ & 0.24 & 0.63 \\
\hline Yes & $12(2.26)$ & $1(1.37)$ & & \\
\hline \multicolumn{5}{|c|}{ Experience SARS personally, $\mathrm{n}(\%)$} \\
\hline No & $308(57.89)$ & $35(47.95)$ & 2.59 & 0.11 \\
\hline Yes & 224(42.11) & $38(52.05)$ & & \\
\hline \multicolumn{5}{|l|}{ Income, n (\%) } \\
\hline$\leq 8$ & $97(18.23)$ & $8(10.96)$ & 2.98 & 0.23 \\
\hline $8-30$ & $352(66.17)$ & $50(68.49)$ & & \\
\hline$\geq 30$ & $83(15.60)$ & $15(20.55)$ & & \\
\hline \multicolumn{5}{|l|}{ Economic loss, n (\%) } \\
\hline$\leq 3$ & 286(53.76) & $37(50.68)$ & 1.48 & 0.69 \\
\hline $3-10$ & $79(14.85)$ & $10(13.70)$ & & \\
\hline$\geq 10$ & 74(13.91) & $9(12.33)$ & & \\
\hline Unknown & $93(17.48)$ & $17(23.29)$ & & \\
\hline \multicolumn{5}{|l|}{ Medical illness, n (\%) } \\
\hline Yes & $123(23.12)$ & 14(19.18) & 0.57 & 0.45 \\
\hline No & $409(76.88)$ & $59(80.82)$ & & \\
\hline Insomnia, Median (IQR) & $5(1-9)$ & $5(1-7)$ & -0.01 & 0.99 \\
\hline Suicide, Median (IQR) & $0(0-0)$ & $0(0-0)$ & -0.95 & 0.34 \\
\hline \multicolumn{5}{|l|}{ Smoking, n (\%) } \\
\hline No & 486(91.35) & $64(87.67)$ & 1.23 & 0.54 \\
\hline Yes & $33(6.20)$ & 7(9.59) & & \\
\hline Smoking cessation & $13(2.44)$ & $2(2.74)$ & & \\
\hline \multicolumn{5}{|l|}{ Drinking, n (\%) } \\
\hline No & $409(76.88)$ & $51(69.86)$ & 1.79 & 0.41 \\
\hline Yes & $110(20.68)$ & $20(27.40)$ & & \\
\hline Abstinence & $13(2.44)$ & $2(2.73)$ & & \\
\hline
\end{tabular}


Multiple logistic regression analysis of SOM-related factors of health care workers.

\begin{tabular}{|lccccc|}
\hline & B & SE & $\boldsymbol{p}$ & OR & $95 \% \mathrm{Cl}$ \\
\hline Sex (ref: Men) & -0.79 & 0.29 & 0.01 & 0.46 & $0.26-0.80$ \\
\hline Working hours per day during COVID-19 outbreak (ref:4-6) & & & 0.01 & & \\
\hline $6-8$ & 2.70 & 1.08 & 0.01 & 14.87 & $1.79-123.78$ \\
\hline $8-10$ & 1.78 & 1.04 & 0.09 & 5.91 & $0.77-45.26$ \\
\hline$\geq 10$ & 2.40 & 1.03 & 0.02 & 11.07 & $1.48-82.75$ \\
\hline Constant & -2.70 & 1.10 & 0.01 & 0.07 & \\
\hline
\end{tabular}

\section{Discussion}

To the best of our knowledge, this was the first study to compare the somatization dimension of SCL-90 between non-medical staff and medical staff during the COVID-19 epidemic. The results showed that: (1) during the epidemic of COVID-19, the prevalence rate of somatization of medical staff was higher than non-medical staff; (2) there were significant differences in the total score of somatization and the scores of each item of somatization between non-medical staff and medical staff; (3) the long daily working hours during the epidemic period of COVID-19 was the risk factor for somatization of medical staff, while the female gender was the protective factor; (4) ethnicity, singleness, insomnia and suicide were the risk factors for somatization of non-medical staff. The results of this study were of great significance to the formulation of psychological support and intervention measures for different populations during the outbreak of COVID-19.

Our findings were consistent with those in one recent study [22] showing that the prevalence rate of somatization in medical staff was higher than that in non-medical staff. Previous studies have shown that somatization refers to the transition from mental state to physical symptoms [23]. Somatic symptoms are defined as a group of physical disorders, such as digestion, appetite, sleep, or physical unhappiness or worry that are not pretending or intentional [24]. These symptoms are or are not caused by organic diseases. An early study suggests that headaches may be associated with the accumulation of adverse psychological effects or the deterioration of their pre-existing medical conditions [16]. Another study shows that the general population has a higher prevalence of depression and anxiety, and they are more likely to develop certain symptoms when experiencing the COVID-19 epidemic, such as cough, chills, dizziness, sore throat and muscle pain [14]. A large number of negative information, including the asymptomatic transmission of the virus carriers and COVID-19, often lead to adverse psychological consequences and may produce a variety of somatic symptoms $[25,26]$.

The real relationship between medical symptoms and psychological stress actually faces enormous challenges, especially in the current tense situation. Before giving a "non-specific" symptom diagnosis, each suspected case needs to urgently rule out any potential possibility [16]. Once the acute infection is solved, psychological support and intervention should be carried out immediately.

The COVID-19 epidemic may bring psychological problems to non-health care workers and health care workers, which may turn into physical symptoms.

Compared with the isolation of the general population, health care workers need to get along with patients face-to-face, working long hours and high intensity, so they are more dangerous and more prone to psychological problems.

In this study, it is found that the total score of SOM and the score of each item of medical staff are higher than those of non-medical staff. Therefore, when people have somatic symptoms, they must carry out psychological intervention after excluding organic diseases.

Our study found that minor ethnicities (non-Han Chinese) were a risk factor. We speculated that there may be some possible reasons.

For example, most ethnic minorities live in remote areas and have relatively poor medical conditions.

According to a previous study, living in rural areas was a risk factor for somatization in the entire sample, as the population in rural areas may worry about infection due to poor medical skills and conditions [22]. COVID-19 is characterized by human-to-human transmission [27, 28], high incidence and potentially lethal [18, 29], which may enhance people's perception of personal danger. With

Page $15 / 18$ 
the increase of COVID-19's confirmed and suspected cases, ordinary people have begun to worry about their health, family health and public health when they are quarantined at home or lost contact with the outside world. In particular, they worry about physical symptoms that may be associated with infection [7], such as cough, dizziness and fever. Further, our study found being single was a protective factor. The possible reason may be that they could not be infected with the coronavirus as long as they ensured their own personal hygiene and did not come into contact with others, when they were alone. A recent study has shown that insomnia may lead to psychological problems related to the epidemic of COVID-19 [17]. When psychological problems cannot be expressed directly, they may be expressed in the form of physical symptoms. Some studies suggest that the highly somatization group had higher suicide attempts and more individual attempts [23]. Isolation can lead to uncomfortable feelings, such as loss of freedom, loneliness from separation from love, and worry about uncertain illness. One study found that when people were quarantined during the previous outbreak, suicides [30] followed. Therefore, in our study, insomnia and suicide were associated with severe physical symptoms in nonhealth care workers.

Our results showed that female gender was a protective factor for health care workers.

The significant differences in personality characteristics (expression and implication) between women and men can partly explain this.

After a short period of training, health care workers were asked to join the front-line battle against COVID-19.

Health care workers were always in contact with infected patients.

Moreover, during the COVID-19 outbreak, health care workers worked continuously under negative pressure for more than 12 hours and were equipped with full-body protection, including protective glasses, double-sided masks, isolation caps, double gloves and foot masks.

To avoid infection when removing protective equipment, health care workers were not allowed to drink water, eat or go to the toilet during working hours.

Some people may develop rashes and cystitis and may even become dehydrated by sweating too much [17].

Under these dangerous conditions, health care workers become mentally and physically exhausted, which can lead to many physical symptoms.

Therefore, our research shows that the long daily working hours during the COVID-19 outbreak was related to the severe somatic symptoms of medical staff.

Our research had three limitations. First, this study was conducted through the self-administered questionnaire of "Wechat" program, which may lead to the deviation of self-choice. Second, the nature of the cross-sectional survey did not reflect causal relationship.

Third, this study lacked follow-up data.

In summary, both non-medical staff and medical staff have somatization symptoms, and the prevalence rate and total score of SOM in medical staff are higher than those in non-medical staff. Factors related to severe somatic symptoms may contribute to the improvement of health policies and the formulation of prevention and treatment intervention strategies.

\section{Declarations}

\section{Acknowledgments}

The authors thank the participants. This research did not receive any specific grant from funding agencies in the public, commercial, or not-for-profit sectors.

\section{Authors' contributions}

XLS: Investigator, participated in study design, data analysis, manuscript writing and review. YJZ: Investigator, participated in study design. WWR: Participated in data analysis, critical revisions for important intellectual content. XYZ: Review of manuscript. All authors read and approved the final version of the manuscript.

\section{Funding}

No.

Availability of data and materials

Page $16 / 18$ 
The datasets generated during and/or analyzed during the current study are available from the corresponding author on request.

\section{Ethics approval and consent to participate}

Informed consent was obtained from the participants. This study protocol was reviewed and approved by the Institute of Psychology, Chinese Academy of Sciences.

\section{Consent for publication}

Not Applicable.

\section{Competing interests}

No conflict of interest was disclosed for each author.

\section{Author details}

${ }^{1}$ Clinical psychology, Yantai Affiliated Hospital of Binzhou Medical University, Yantai, China; ${ }^{2}$ Shenzhen Kangning Hospital, Shenzhen, China; ${ }^{3}$ Unit of psychiatry, Faculty of Health Sciences, University of Macau, Macau SAR, China; ${ }^{4}$ CAS Key Laboratory of Mental Health, Institute of Psychology, Chinese Academy of Sciences, Beijing, China.

\section{Abbreviations}

SOM: somatization; COVID-19: The coronavirus disease 2019; WHO: the World Health Organization; PHEIC: Public Health Emergency of International Concern; PTSD: posttraumatic stress disorder; IES-R: the Impact of Events Scale-Revised; DASS-21: Depression Anxiety Stress Scales; ISI: the Insomnia Severity Index; MINI: Mini International Neuropsychiatric Interview; OC: Obsessive-Compulsive; IS: Interpersonal Sensitivity; DEP: Depression; ANX: Anxiety; HOS: Hostility; PHOB: Phobic Anxiety; PAR: Paranoid Ideation; PSY: Psychoticism; IQRs: the interquartile ranges; BMI: body mass index

\section{References}

1. Qiu J, Shen B, Zhao M, Wang Z, Xie B, Xu Y: A nationwide survey of psychological distress among Chinese people in the COVID19 epidemic: implications and policy recommendations. Gen Psychiatr 2020, 33(2):e100213.

2. Tian F, Li H, Tian S, Yang J, Shao J, Tian C: Psychological symptoms of ordinary Chinese citizens based on SCL-90 during the level I emergency response to COVID-19. Psychiatry Res 2020, 288:112992.

3. Bao Y, Sun Y, Meng S, Shi J, Lu L: 2019-nCoV epidemic: address mental health care to empower society. The Lancet 2020, 395(10224):e37-e38.

4. Ebrahim SH, Ahmed QA, Gozzer E, Schlagenhauf P, Memish ZA: Covid-19 and community mitigation strategies in a pandemic. BMJ 2020, 368:m1066.

5. Yezli S, Khan A: COVID-19 social distancing in the Kingdom of Saudi Arabia: Bold measures in the face of political, economic, social and religious challenges. Travel Med Infect Dis 2020:101692.

6. Wilder-Smith A, Freedman DO: Isolation, quarantine, social distancing and community containment: pivotal role for old-style public health measures in the novel coronavirus (2019-nCoV) outbreak. J Travel Med 2020, 27(2).

7. Desclaux A, Badji D, Ndione AG, Sow K: Accepted monitoring or endured quarantine? Ebola contacts' perceptions in Senegal. Soc Sci Med 2017, 178:38-45.

8. Reynolds DL, Garay JR, Deamond SL, Moran MK, Gold W, Styra R: Understanding, compliance and psychological impact of the SARS quarantine experience. Epidemiology and Infection 2007, 136(7):997-1007.

9. Wilken JA, Pordell P, Goode B, Jarteh R, Miller Z, Saygar BG, Maximore L, Borbor WM, Carmue M, Walker GW et al: Knowledge, Attitudes, and Practices among Members of Households Actively Monitored or Quarantined to Prevent Transmission of Ebola Virus Disease - Margibi County, Liberia: February-March 2015. Prehosp Disaster Med 2017, 32(6):673-678.

10. Brooks SK, Webster RK, Smith LE, Woodland L, Wessely S, Greenberg N, Rubin GJ: The psychological impact of quarantine and how to reduce it: rapid review of the evidence. The Lancet 2020, 395(10227):912-920. 
11. Shultz JM, Baingana F, Neria Y: The 2014 Ebola outbreak and mental health: current status and recommended response. JAMA 2015, 313(6):567-568.

12. Kamara S, Walder A, Duncan J, Kabbedijk A, Hughes P, Muana A: Mental health care during the Ebola virus disease outbreak in Sierra Leone. Bull World Health Organ 2017, 95(12):842-847.

13. Otte S, Vasic N, Nigel S, Streb J, Ross T, Spitzer C, Grabe HJ, Dudeck M: Different yet similar? Prisoners versus psychiatric patients - A comparison of their mental health. Eur Psychiatry 2017, 44:97-103.

14. Wang C, Pan R, Wan X, Tan Y, Xu L, Ho CS, Ho RC: Immediate Psychological Responses and Associated Factors during the Initial Stage of the 2019 Coronavirus Disease (COVID-19) Epidemic among the General Population in China. Int J Environ Res Public Health 2020, 17(5).

15. Yang Y, Li W, Zhang Q, Zhang L, Cheung T, Xiang Y-T: Mental health services for older adults in China during the COVID-19 outbreak. The Lancet Psychiatry 2020, 7(4):e19.

16. Chew NWS, Lee GKH, Tan BYQ, Jing M, Goh Y, Ngiam NJH, Yeo LLL, Ahmad A, Ahmed Khan F, Napolean Shanmugam G et al: A multinational, multicentre study on the psychological outcomes and associated physical symptoms amongst healthcare workers during COVID-19 outbreak. Brain Behav Immun 2020.

17. Zhang C, Yang L, Liu S, Ma S, Wang Y, Cai Z, Du H, Li R, Kang L, Su M et al: Survey of Insomnia and Related Social Psychological Factors Among Medical Staff Involved in the 2019 Novel Coronavirus Disease Outbreak. Front Psychiatry 2020, 11:306.

18. Lai J, Ma S, Wang Y, Cai Z, Hu J, Wei N, Wu J, Du H, Chen T, Li R et al: Factors Associated With Mental Health Outcomes Among Health Care Workers Exposed to Coronavirus Disease 2019. JAMA Netw Open 2020, 3(3):e203976.

19. Yu Y, Wan C, Huebner ES, Zhao X, Zeng W, Shang L: Psychometric properties of the symptom check list 90 (SCL-90) for Chinese undergraduate students. J Ment Health 2019, 28(2):213-219.

20. Thorndike FP, Ritterband LM, Saylor DK, Magee JC, Gonder-Frederick LA, Morin CM: Validation of the Insomnia Severity Index as a Web-Based Measure. Behavioral Sleep Medicine 2011, 9(4):216-223.

21. Sheehan DV, Lecrubier Y, Sheehan KH, Amorim P, Janavs J, Weiller E, Hergueta T, Baker R, Dunbar GC: The Mini-International Neuropsychiatric Interview (M.I.N.I.): the development and validation of a structured diagnostic psychiatric interview for DSM-IV and ICD-10. J Clin Psychiatry 1998, 59 Suppl 20:22-33;quiz 34-57.

22. Zhang WR, Wang K, Yin L, Zhao WF, Xue Q, Peng M, Min BQ, Tian Q, Leng HX, Du JL et al: Mental Health and Psychosocial Problems of Medical Health Workers during the COVID-19 Epidemic in China. Psychother Psychosom 2020:1-9.

23. Edgcomb JB, Kerner B: Predictors and outcomes of somatization in bipolar I disorder. A latent class mixture modeling approach. J Affect Disord 2018, 227:681-687.

24. Kapfhammer HP: [Depressive, anxiety and posttraumatic stress disorders as long-term sequelae of intensive care treatment]. Nervenarzt 2016, 87(3):253-263.

25. Adams JG, Walls RM: Supporting the Health Care Workforce During the COVID-19 Global Epidemic. JAMA 2020.

26. Joob B, Wiwanitkit V: Traumatization in medical staff helping with COVID-19 control. Brain Behav Immun 2020.

27. Li Q, Guan X, Wu P, Wang X, Zhou L, Tong Y, Ren R, Leung KSM, Lau EHY, Wong JY et al: Early Transmission Dynamics in Wuhan, China, of Novel Coronavirus-Infected Pneumonia. N Engl J Med 2020, 382(13):1199-1207.

28. Zhu N, Zhang D, Wang W, Li X, Yang B, Song J, Zhao X, Huang B, Shi W, Lu R et al: A Novel Coronavirus from Patients with Pneumonia in China, 2019. N Engl J Med 2020, 382(8):727-733.

29. Wang W, Tang J, Wei F: Updated understanding of the outbreak of 2019 novel coronavirus (2019-nCoV) in Wuhan, China. J Med Virol 2020, 92(4):441-447.

30. Barbisch D, Koenig KL, Shih FY: Is There a Case for Quarantine? Perspectives from SARS to Ebola. Disaster Med Public Health Prep 2015, 9(5):547-553. 